\title{
Fresh Produce Safety and Quality: Chlorine Dioxide's Role
}

\author{
Siva Kumar Malka and Me-Hea Park* \\ Postharvest Research Division, National Institute of Horticultural and Herbal Science, Wanju-gun, South Korea
}

\section{OPEN ACCESS}

Edited by:

María Serrano,

Miguel Hernández University of Elche,

Spain

Reviewed by:

Mark Morgan,

The University of Tennessee,

Knoxville, United States

Charu Gupta,

Amity University, India

Maria Concetta Strano,

Council for Agricultural

and Economics Research (CREA),

Italy

*Correspondence:

Me-Hea Park

poemmich@korea.kr

Specialty section:

This article was submitted to

Crop and Product Physiology, a section of the journal

Frontiers in Plant Science

Received: 14 September 2021

Accepted: 16 December 2021

Published: 11 January 2022

Citation:

Malka SK and Park M-H (2022) Fresh Produce Safety and Quality:

Chlorine Dioxide's Role.

Front. Plant Sci. 12:775629.

doi: 10.3389/fpls.2021.775629
Maintaining microbial safety and quality of fresh fruits and vegetables are a global concern. Harmful microbes can contaminate fresh produce at any stage from farm to fork. Microbial contamination can affect the quality and shelf-life of fresh produce, and the consumption of contaminated food can cause foodborne illnesses. Additionally, there has been an increased emphasis on the freshness and appearance of fresh produce by modern consumers. Hence, disinfection methods that not only reduce microbial load but also preserve the quality of fresh produce are required. Chlorine dioxide $\left(\mathrm{ClO}_{2}\right)$ has emerged as a better alternative to chlorine-based disinfectants. In this review, we discuss the efficacy of gaseous and aqueous $\mathrm{ClO}_{2}$ in inhibiting microbial growth immediately after treatment (short-term effect) versus regulating microbial growth during storage of fresh produce (long-term effect). We further elaborate upon the effects of $\mathrm{ClO}_{2}$ application on retaining or enhancing the quality of fresh produce and discuss the current understanding of the mode of action of $\mathrm{ClO}_{2}$ against microbes affecting fresh produce.

Keywords: chlorine dioxide, fresh produce, microbial safety, log reduction, produce quality, storage, shelf life, antimicrobial mechanism

\section{INTRODUCTION}

Fresh produce, including fruits and vegetables, is a good source of nutrients and an important component of a healthy and balanced diet. However, fresh produce is susceptible to microbial contamination, which may occur at any step of the food supply chain, from sowing the crop to delivering it to the customer. Furthermore, cross-contamination can occur during processing, packaging, or transporting fresh produce. Most common sources of food contamination are soil, animal manure, and irrigation water, and consuming contaminated food may lead to the outbreak of foodborne illnesses. In the United States of America, 340 foodborne outbreaks, from 2009 to 2018, were associated with fresh produce (CDC, 2018). Furthermore, 5,175 foodborne outbreaks were reported in Europe in 2019, involving 49,463 cases, 3,859 hospitalizations, and 60 fatalities (European Food Safety Authority, 2021). Foodborne diseases not only affect human health but also pose challenges to tourism, agricultural, and food industries, thereby seriously affecting socioeconomic development (WHO, 2015).

Microbial contamination can affect the quality and shelf-life of fresh produce. In 2010, an estimated 31\% of the total food produce worth $\$ 161.6$ billion was declared unfit for human consumption at the retail and consumer levels (Buzby et al., 2014). With an increased awareness of the importance of fresh produce consumption for a healthy lifestyle, the concerns of modern

Abbreviations: MAP, modified atmosphere packaging; PPO, polyphenol oxidase; PPM, parts per million. 
consumers regarding the freshness, appearance, and microbial safety of fresh produce have increased. At the retail level, primarily in supermarkets and hypermarkets, $15-30 \%$ of the fresh produce was rejected by consumers because of quality standards that over-emphasize appearance (FAO, 2011). Hence, microbial safety and high quality have emerged as a concern for the food industry and consumers.

Several physical and chemical disinfection methods have been used to reduce the microbial load on fresh produce (Deng et al., 2020; Chacha et al., 2021). However, a potent disinfection method must fulfill the following criteria: high efficacy against pathogens, ability to reduce microbial spoilage, potential to retain nutritional quality, no formation of intolerable levels of human toxic by-products or residues, and no environmental impact (Joshi et al., 2013). The efficacies of various physical disinfection methods, including high hydrostatic pressure, cold plasma, ultraviolet, ultrasound, pulsed, and ionizing radiation, have been examined. However, these methods have various disadvantages. For instance, ultrasound shows limited antimicrobial effect, ultraviolet light has low penetration and shade effect from complex surface properties of produce affects its efficiency, and pulsed light increases temperature that deteriorates the quality of treated produce (Deng et al., 2020).

Chlorine is the most commonly used chemical disinfectant in the food industry, which is effective against a broad range of pathogens and whose efficacy has been evaluated in a wide variety of fresh produce (Praeger et al., 2018). However, chlorine may react with natural organic matter and form halogenated byproducts, such as trihalomethanes or haloacetic acids (Praeger et al., 2018). These by-products are carcinogenic and not environment-friendly. Moreover, owing to safety and efficacy concerns, the use of chlorine for the sterilization of freshcut produce has been banned in countries such as Belgium, Switzerland, and Netherlands (Deng et al., 2020). Therefore, several chemical alternatives, such as chlorine dioxide $\left(\mathrm{ClO}_{2}\right)$, ozone, electrolyzed water, essential oils, high-pressure carbon dioxide, and organic acids, have been identified or proposed (Deng et al., 2020). For instance, electrolyzed water, and ozone are potent disinfectants; however, for the effective microbial reduction high concentration or prolonged exposure is required. Excessive usage of these treatments can negatively affect produce quality (Deng et al., 2020). Organic acids are safe and easy to use but their antimicrobial efficiency is limited. Essential oils are natural antimicrobial agents; however, it is practically difficult use these oils because of their hydrophobic, volatile and unstable nature (Deng et al., 2020).

Application of $\mathrm{ClO}_{2}$, an oxidative gas, is effective in controlling the bacterial, fungal, and viral contamination of fresh produce (Praeger et al., 2018; Sun et al., 2019). In contrast to chlorine, $\mathrm{ClO}_{2}$ neither produces toxic by-products nor does it alter the nutritive and organoleptic qualities of food products, and is effective over a wide $\mathrm{pH}$ range ( $\mathrm{pH} 3-8)$. In addition, it is widely used as a bleaching agent in paper industry and as a disinfectant in laboratories, hospitals, public places, and other areas (Praeger et al., 2018). Owing to its efficacy and safety, $\mathrm{ClO}_{2}$ has been approved for the disinfection of fresh produce and in food processing industries (FDA, 2008). Recently, Praeger et al. (2018) and Sun et al. (2019) comprehensively reviewed antimicrobial activity of aqueous and gaseous $\mathrm{ClO}_{2}$, respectively. This review focuses on the effects of $\mathrm{ClO}_{2}$ application on the initial reduction in microbial growth (short-term effect) and the final reduction in microbial populations during the storage of fresh produce (longterm effect). We further discuss the efficacy of $\mathrm{ClO}_{2}$ application in maintaining the quality of fresh produce and the action mechanism of $\mathrm{ClO}_{2}$ against microbes affecting fresh produce.

\section{MODES OF CHLORINE DIOXIDE APPLICATION}

Chlorine dioxide is a yellowish-green gas and is highly water soluble, approximately 10 times more soluble in water than chlorine, particularly in cold water. Moreover, it remains in solution as a dissolved gas without hydrolyzing. Hence, it can be used in aqueous as well as gaseous forms. The advantages and limitations of using aqueous and gaseous $\mathrm{ClO}_{2}$ are summarized in Table 1.

Aqueous $\mathrm{ClO}_{2}$ solution can be used to spray, immerse, or wash fresh produce, as it ensures adequate $\mathrm{ClO}_{2}$ concentration and contact time, both of which are the determinants of its efficacy against pathogens. Moreover, aqueous $\mathrm{ClO}_{2}$ application is relatively easy to implement or adopt in the existing washing lines in food industries without modifying subsequent processes (Wu and Kim, 2007). However, water rinsing, an additional step, is required following aqueous $\mathrm{ClO}_{2}$ treatment, resulting in residual moisture on the produce surface that may stimulate microbial growth (Trinetta et al., 2011).

TABLE 1 | Chlorine dioxide $\left(\mathrm{ClO}_{2}\right)$ application in aqueous and gaseous form: advantages and disadvantages.

\begin{tabular}{ll}
\hline Aqueous application & Gaseous application \\
\hline Advantages (Praeger et al., 2018) & Advantages (Sun et al., 2019) \\
Easy to handle, inexpensive & Higher antimicrobial activity \\
It can be used in the form of spray, & It can be applied as batch treatment or \\
immerse or washing & continuous treatment \\
Concentration and contact can be & High accessibility to microbes \\
maintained & irrespective of surface barriers \\
Easy to adopt in industrial washing lines & No water rinsing required after the \\
& treatment \\
& It can impact microbial internalization \\
& No issue of cross-contamination of \\
wisadvantages (Praeger et al., 2018) & Disadvantages (Sun et al., 2019) \\
$\begin{array}{ll}\text { Produce surface properties can affect } \\
\text { ClO } 2 \text { accessibility to microbes }\end{array}$ & Needs onsite generation \\
Cross-contamination of wash water & Needs technical knowledge \\
$\begin{array}{l}\text { Water rinsing is required after the } \\
\text { treatment }\end{array}$ & laborious to perform, expensive \\
Residual moisture after the water & Explosive at higher concentration \\
rinsing can promote microbial growth & \\
Not suitable for dried foods & Challenging to maintain concentration \\
Relatively less effect on microbial & and contact time \\
Difficult to implement at industry scale
\end{tabular}
internalization 
In contrast to aqueous $\mathrm{ClO}_{2}$, gaseous $\mathrm{ClO}_{2}$ is more effective against pathogens because of its higher potential to reach microbes irrespective of the surface irregularities of fresh produce (Han et al., 2001a). $\mathrm{ClO}_{2}$ is generally produced by the reaction of an acid with sodium chlorate or sodium chlorite and chlorine gas (Praeger et al., 2018). As gaseous $\mathrm{ClO}_{2}$ application does not require water, the risk of cross-contamination with recycled wash-water can be avoided. However, the major limitation of gaseous $\mathrm{ClO}_{2}$ application is its on-site production, as it cannot be compressed and stored or transported under pressure (EPA, 1999). Moreover, $\mathrm{ClO}_{2}$ production is laborious and expensive, and it is technically challenging to maintain a precise $\mathrm{ClO}_{2}$ concentration during gaseous treatment (Wu and Kim, 2007).

Alternatively, several packaging systems that can generate and release $\mathrm{ClO}_{2}$ have been developed. In these systems, materials that generate gaseous $\mathrm{ClO}_{2}$, including perforated sachets, pouches, tablets, films, and pads, are incorporated into the packaging system using different methods (Singh et al., 2021). Furthermore, for the development of an active packing material, factors, such as the release rate of the active material, its efficacy against microbes, and the maintenance of shelf-life of the product to be packed, are taken into consideration. These packaging systems are often designed to be used in combination with other technologies, such as modified atmosphere packaging (MAP).

\section{MODES OF CHLORINE DIOXIDE ACTION}

\section{Antimicrobial Mechanisms}

The antibacterial mechanism of $\mathrm{ClO}_{2}$ includes destabilization of the cell membrane, alteration of membrane permeability, and interruption of protein synthesis (Figure $\mathbf{1 A}$ ). $\mathrm{ClO}_{2}$ reacts with oxygenated compounds and proteins in cell membranes, resulting in the disruption of cell metabolism (Praeger et al., 2018). Membrane damage in $\mathrm{ClO}_{2}$-exposed Bacillus subtilis spores inhibits their development after germination (Young and Setlow, 2003). Moreover, $\mathrm{ClO}_{2}$ oxidizes the exposed sulfhydryl groups of cell surface proteins, thereby causing membrane damage and increasing outer membrane permeability. Loss of permeability control, evident from the efflux of $\mathrm{K}^{+}$ions, results in the destruction of transmembrane ionic gradient in Escherichia coli (Berg et al., 1986). Furthermore, loss of cell activity or cell death in $\mathrm{ClO}_{2}$-treated Pseudomonas aeruginosa and Staphylococcus aureus is correlated with the increased permeability of inner and outer cell membranes and the subsequent release of vital nuclear materials (Ofori et al., 2018). At higher concentrations, $\mathrm{ClO}_{2}$ induces accumulation of malondialdehyde (MDA) content, indicating the occurrence of membrane peroxidation (Bridges et al., 2020). However, previous studies based on transmission electron microscopy did not reveal significant morphological damage or cell lysis (Ofori et al., 2018; Bridges et al., 2020). Additionally, amino acids, including cysteine, tyrosine, tryptophan, histidine, and proline, are responsive to $\mathrm{ClO}_{2}$, with their order of reactivity from high to low, respectively (Sharma and Sohn, 2012).

The virucidal mechanism of $\mathrm{ClO}_{2}$ varies depending on the composition and three-dimensional structure of viral proteins and nucleic acids (Figure 1B). Degradation of viral capsid proteins inhibits the attachment of $\mathrm{ClO}_{2}$-exposed bacteriophages to host cells ( $\mathrm{Ge}$ et al., 2021). Similarly, the $\mathrm{ClO}_{2}$-mediated destruction of glycoproteins affects viral attachment to cell receptors and alters the life cycle of porcine reproductive and respiratory syndrome virus (Zhu et al., 2019). In addition, the denaturation of viral proteins has been reported to be involved

A

\section{Cell membrane}

- Destabilization of cell membrane

DNA

- Oxidation of DNA

Protein

- Oxidation of amino acids/proteins

- Inhibition of protein synthesis

B

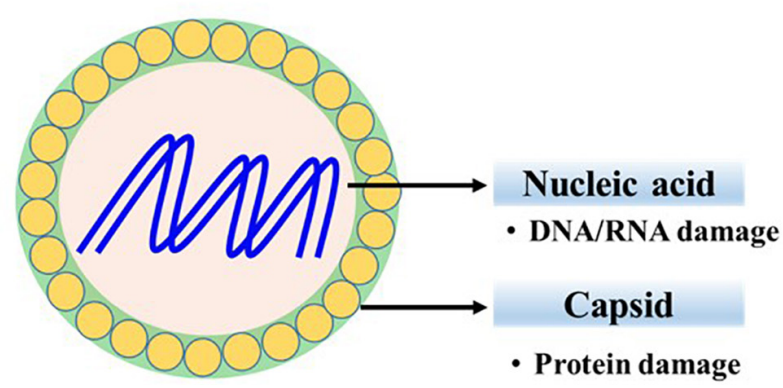

FIGURE 1 | Mechanisms of chlorine dioxide against bacteria (A) and viruses (B). 
in the inactivation of human rotavirus (Xue et al., 2013). $\mathrm{ClO}_{2}$ damages the 5' non-coding region in the viral genome that is necessary for formation of new virus particles within the host cells (Li et al., 2004; Jin et al., 2013). Furthermore, RNA damage, in addition to protein damage, has been attributed to the inactivation of poliovirus (Simonet and Gantzer, 2006).

The fungicidal mechanism of $\mathrm{ClO}_{2}$ involves disruption of both the plasma and mitochondrial membranes (Zhang and $\mathrm{Fu}, 2018$; Lin et al., 2021). $\mathrm{ClO}_{2}$ treatment causes ion leakage, inhibition of key enzyme activities in metabolic pathways, and alteration of cell structure in Saccharomyces cerevisiae (Zhu et al., 2013). Further, $\mathrm{ClO}_{2}$ induces membrane lipid peroxidation, which is evident by enhanced MDA levels in Penicillum expansum (Zhang and $\mathrm{Fu}, 2018$ ).

\section{Potential Mechanisms Regulating Fresh Produce Quality}

The mechanisms underlying the regulation of fresh produce quality by $\mathrm{ClO}_{2}$ include its impact on respiration rate and ethylene biosynthesis (Figure 2). $\mathrm{ClO}_{2}$-mediated inhibition of ethylene biosynthesis, brought about by the suppression of ethylene biosynthesis-related genes, including ACS2, ACO1, and ACO3 (Guo et al., 2013, 2014), alters the physiological and biochemical changes that occur during fruit maturation and senescence. Reduced respiration rate and transpiration delay the consumption of nutrients and water, which directly influences fruit firmness, mass loss, and softening (Chen and Zhu, 2011; Guo et al., 2013, 2014). The quality of fresh produce during storage depends on the correlation between cellular energy and redox status. For example, delayed senescence in $\mathrm{ClO}_{2}$-treated longan fruit has been reported to be associated with an altered redox state and increased cellular energy (Chumyam et al., 2016). Moreover, reduced microbial incidence in $\mathrm{ClO}_{2}$ treated produce leads to quality retention and shelf-life extension (Islam et al., 2017).

\section{EFFECTS OF CHLORINE DIOXIDE TREATMENT ON MICROBES AFFECTING FRESH PRODUCE}

Chlorine dioxide concentration and contact time are crucial in determining the efficacy of $\mathrm{ClO}_{2}$, which may also vary with the type of microorganism and fresh produce. The short-term and long-term efficacy of $\mathrm{ClO}_{2}$ in inhibiting the growth of preexisting or artificially inoculated microorganisms have been demonstrated in a wide variety of fresh produce (Tables 2, 3). Furthermore, some reports suggested that artificially inoculated human pathogens, such as E. coli, Salmonella spp., and Listeria monocytogenes, exhibit higher inactivation on fresh produce than natural microflora after $\mathrm{ClO}_{2}$ application (Praeger et al., 2018).

\section{Short-Term Effects Vegetables}

Washing leafy vegetables with aqueous $\mathrm{ClO}_{2}$ was effective in inactivating natural microflora. For instance, the initial populations of aerobic mesophilic, aerobic psychrotrophic, and lactic acid bacteria, yeast, and molds, in raw asparagus lettuce slices decreased by 1-3 log upon treatment with $100 \mathrm{ppm}$ $\mathrm{ClO}_{2}$ for 20 min (Chen et al., 2010). Furthermore, exposure to $3 \mathrm{ppm} \mathrm{ClO}_{2}$ for $1 \mathrm{~min}$ reduced epiphytic microbiota on fresh-cut iceberg lettuce by 1-2 log (López-Gálvez et al., 2010). The efficacy of $\mathrm{ClO}_{2}$ against artificially inoculated pathogens, such as E. coli, Salmonella spp., and L. monocytogenes, has been extensively investigated in lettuce. A 2 min-long treatment with 100 or 200 ppm aqueous $\mathrm{ClO}_{2}$ in iceberg lettuce resulted in $>1 \log$ reduction in E. coli O157:H7 load (Keskinen et al., 2009). Similar results were obtained for S. typhimurium and L. monocytogenes inoculated on iceberg lettuce with lower $\mathrm{ClO}_{2}$ concentrations but longer exposure (10 min) (Kim et al., 2008). Moreover, Rodgers et al. (2004) observed >5 log reduction in the loads of E. coli O157:H7 and L. monocytogenes after $\mathrm{ClO}_{2}$ application ( $5 \mathrm{ppm}$ for $5 \mathrm{~min}$ ) on green leaf lettuce. Similar observations were made in whole heads of iceberg lettuce exposed to $5 \mathrm{ppm}$ gaseous $\mathrm{ClO}_{2}$ for $15-20 \mathrm{~min}$ (Mahmoud and Linton, 2008). In spinach leaves, treatment with high $\mathrm{ClO}_{2}$ concentration for a short contact time (100 ppm for $5 \mathrm{~min}$ ) or low $\mathrm{ClO}_{2}$ concentration with a long exposure time (10 ppm for $20 \mathrm{~min}$ ) yielded approximately similar levels of pathogen reduction (Lee and Baek, 2008; Park and Kang, 2015b).

Several studies have investigated the disinfection of tomatoes using $\mathrm{ClO}_{2}$ (Praeger et al., 2018; Sun et al., 2019). For artificially inoculated human pathogens, 5-7 log reduction in microbial load has been observed with gaseous $\mathrm{ClO}_{2}$ concentrations $<1$ ppm (Bhagat et al., 2010; Olanya et al., 2015; Netramai et al., 2016). For instance, a $12 \mathrm{~min}$ exposure to $0.5 \mathrm{ppm} \mathrm{ClO}_{2}$ resulted in $>5 \mathrm{log}$ reduction in Salmonella and L. monocytogenes loads in hydroponically grown tomatoes (Bhagat et al., 2010). With an increased exposure time (approximately $1 \mathrm{~h}$ ), grape tomatoes exhibited $>7 \mathrm{log}$ reduction in the load of Salmonella spp., at $25^{\circ} \mathrm{C}$ (Netramai et al., 2016). Trinetta et al. (2010) evaluated the efficacy of short-term exposure of high $\mathrm{ClO}_{2}$ concentrations in the inactivation of $S$. enterica inoculated on tomatoes and observed that the initial populations $(6 \mathrm{log})$ were reduced to $3 \log , 2 \log$, and $1 \log$ in response to $8 \mathrm{ppm} \mathrm{ClO}_{2}$ for $60 \mathrm{~s}$, $10 \mathrm{ppm} \mathrm{ClO}_{2}$ for $120 \mathrm{~s}$, and $10 \mathrm{ppm}$ for $180 \mathrm{~s}$, respectively. Previous studies suggest that the disinfection efficiency of $\mathrm{ClO}_{2}$ on tomatoes freshly spot-inoculated with Salmonella and Erwinia carotovora is higher than that on produce with desiccated inoculum (Pao et al., 2007). Moreover, tomato packaging with $\mathrm{ClO}_{2}$-generating materials, such as films, sachets, and pouches, is effective in achieving microbial reduction from 4 to $6 \log$ to undetectable levels (Mahovic et al., 2007; Ray et al., 2013; Sun et al., 2017b; Zhou et al., 2018). Additionally, Trinetta et al. (2013) reported complete inhibition of the mycelial growth of Alternaria alternate and Stemphylium vesicarium using a 3 minlong $\mathrm{ClO}_{2}$ treatment. $\mathrm{ClO}_{2}$ efficiency has also been reported to increase with an increase in relative humidity and temperature (Park and Kang, 2015b, 2018).

Antimicrobial efficiency of $\mathrm{ClO}_{2}$ has also been evaluated in other fresh vegetables. After $\mathrm{ClO}_{2}$ treatment, minimally processed carrots exhibited significantly decreased levels of mesophilic aerobic bacteria (1.9 log), psychrotrophs (1.7 $\log )$, lactic acid bacteria (2.6 log), and yeast $\left(\begin{array}{lll}0.7 & \log \end{array}\right)$ 


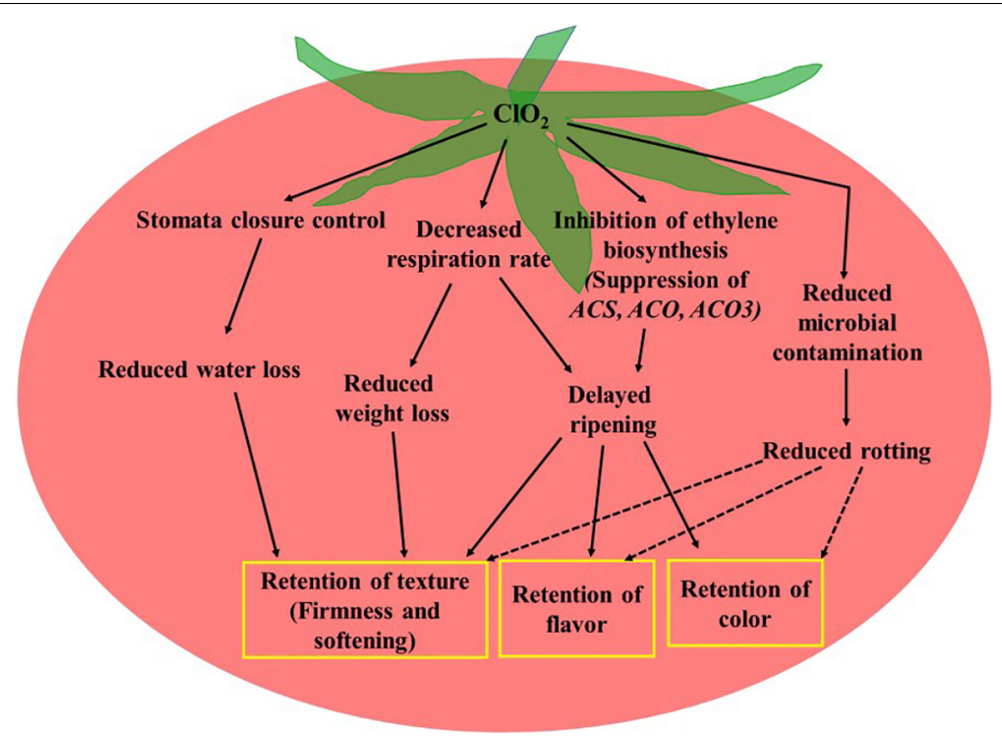

FIGURE 2 | Potential mechanisms of chlorine dioxide regulating fresh produce quality. Dashed arrows indicate indirect effect.

(Gomez-Lopez et al., 2007). Potato exposed to $\mathrm{ClO}_{2}$ for $5 \mathrm{~h}$ exhibited a $5 \log$ and $6 \log$ reduction in natural microflora and Pseudomonas aeruginosa, respectively (Wu and Rioux, 2010). $\mathrm{ClO}_{2}$ application for $30 \mathrm{~min}$ reduced the loads of $E$. coli O157:H7 or L. monocytogenes inoculated on surface-injured green peppers by 6.5 and $3.5 \mathrm{log}$, respectively (Han et al., 2000, 2001b). Similarly, $\mathrm{ClO}_{2}$ treatment effectively inactivated natural microbiota and inoculated Salmonella on the surface of chili peppers (Lee et al., 2018). Furthermore, disinfection of red chili pepper with $\mathrm{ClO}_{2}$ after hot-air drying significantly decreased Bacillus cereus spore populations below the detection limit (1.7 log) (Kim et al., 2017).

\section{Fruits}

Aqueous $\mathrm{ClO}_{2}$ treatment (80 ppm for $15 \mathrm{~min}$ ) yielded an approximately $1.5-3 \log$ reduction in aerobic bacteria in mulberry (Chen et al., 2011). Similarly, a $10 \mathrm{~min}$ exposure to 100 ppm $\mathrm{ClO}_{2}$ significantly decreased the initial populations of natural microflora in blueberries (Chun et al., 2013). With gaseous $\mathrm{ClO}_{2}$ application (5.5 ppm), > 5 log reduction in the load of artificially inoculated Salmonella spp., was observed in whole blueberries and strawberries (Annous et al., 2020). Similar results were observed for L. monocytogenes, E. coli O157:H7, yeast, and molds (Mahmoud et al., 2007; Popa et al., 2007; Wu and Kim, 2007). However, $\mathrm{ClO}_{2}$ efficacy was higher for Salmonella inoculated on blueberry skin tissues than for those inoculated on stem scar tissues (Sy et al., 2005a). Sun et al. (2014) reported an approximately $4 \mathrm{log}$ reduction in the load of Colletotrichum acutatum on blueberries with $\mathrm{ClO}_{2}$ fumigation. Berries treated with $\mathrm{ClO}_{2}$, generated in a small chamber with acidified sodium chlorite solution, reduced Tulane virus populations by $>1-3.3$ log (Kingsley et al., 2018; Kingsley and Annous, 2019).

Previous studies have investigated the effects of various concentrations and exposure times of $\mathrm{ClO}_{2}$ on the populations of E. coli O157:H7 and L. monocytogenes on the skin surface, stem, and calyx cavities of apples. Although an exposure of $4.0 \mathrm{ppm} \mathrm{ClO}_{2}$ for $10 \mathrm{~min}$ resulted in a $5.5 \mathrm{log}$ reduction in L. monocytogenes populations, treatment with $12.0 \mathrm{ppm} \mathrm{ClO}_{2}$ for $10 \mathrm{~min}, 7.2 \mathrm{ppm} \mathrm{ClO}_{2}$ for $20 \mathrm{~min}$, or $4.8 \mathrm{ppm} \mathrm{ClO}_{2}$ for 30 min completely suppressed the bacterial population, which was initially inoculated on the skin (Du et al., 2002, 2003). Moreover, after $3 \mathrm{~h}$ of exposure to low $\mathrm{ClO}_{2}$-releasing sachets, the population of Alicyclobacillus acidoterrestris spores decreased to $4.5 \mathrm{log}$ on apple surface (Lee et al., 2006). A 10 min-long fumigation with $0.5 \mathrm{ppm} \mathrm{ClO}_{2}$ on oranges resulted in $>5 \mathrm{log}$ reduction in Salmonella load (Bhagat et al., 2011). Gaseous $\mathrm{ClO}_{2}$, at concentrations $200-1,800 \mathrm{ppm}$, significantly lowered the incidence of green mold on citrus fruits, including kumquats, mandarins, and Peru oranges, and Penicillium digitatum on grapefruits (Liu et al., 2020). Furthermore, $\mathrm{ClO}_{2}$ treatment effectively reduced Xanthomonas citri contamination in both artificially and naturally contaminated citrus fruits (Behlau et al., 2021). Reports suggest that $\mathrm{ClO}_{2}$ treatment is more effective against $E$. coli inoculated on smooth non-stem-scar surfaces than on rough stem-scar areas (Pao and Davis, 1999). Additionally, $X$. citri on grapefruit surface requires a higher $\mathrm{ClO}_{2}$ concentration for complete inactivation than E. coli (Sun et al., 2017a).

\section{Long-Term Effects}

Postharvest storage is essential for some types of fresh produce; however, microbial populations gradually increase during their storage. Hence, a strong disinfection method is required to ensure long-term protection of the treated produce. An initial reduction of microbial load is important for extending the microbiological shelf-life of fresh produce (Lin et al., 2021). Previous studies have reported different efficacies of $\mathrm{ClO}_{2}$ in inhibiting microbial growth during postharvest storage of produce (Praeger et al., 2018; Singh et al., 2021). A 20 min exposure to aqueous $\mathrm{ClO}_{2}$ inhibited the growth of natural microflora and prolonged 
TABLE 2 | Effects of chlorine dioxide on short- and long-term reduction of microorganisms in vegetables.

\begin{tabular}{|c|c|c|c|c|c|c|c|c|}
\hline \multirow[t]{2}{*}{ Produce } & \multirow[t]{2}{*}{ Microorganism } & \multicolumn{3}{|c|}{ Treatment conditions } & \multicolumn{2}{|c|}{ Log reductions } & \multirow[t]{2}{*}{ Storage conditions } & \multirow[t]{2}{*}{ References } \\
\hline & & Mode & Concentration & Duration & Short-term & Long-term & & \\
\hline \multirow[t]{3}{*}{ Lettuce } & Total aerobic bacteria & $\mathrm{Aq}$ & 50 ppm & $10 \min$ & 1.77 & 0.9 & 8 days, $4^{\circ} \mathrm{C}$ & Kim et al., 2007 \\
\hline & Yeasts and molds & & & & 1.34 & 1.16 & & \\
\hline & Coliforms & & & & 1.1 & 0.9 & & \\
\hline \multirow[t]{3}{*}{ Lettuce } & Escherichia coli O157:H7 & $\mathrm{Aq}$ & 20 ppm & $10 \min$ & 1.44 & 1.38 & 4 days, $4^{\circ} \mathrm{C}$ & Kim et al., 2008 \\
\hline & Salmonella & & & & 1.95 & 1.91 & & \\
\hline & Listeria monocytogenes & & & & 1.2 & 0.99 & & \\
\hline Lettuce & $\begin{array}{c}\text { Escherichia coli O157:H7, L. } \\
\text { monocytogenes }\end{array}$ & $\mathrm{Aq}$ & 3, 5 ppm & $5 \min$ & 5.6 & Unchanged & 9 days, $4^{\circ} \mathrm{C}$ & $\begin{array}{l}\text { Rodgers et al., } \\
2004\end{array}$ \\
\hline \multirow[t]{5}{*}{ Lettuce } & Escherichia coli O157:H7 & $\mathrm{G}$ & 5 ppm & $10 \min$ & 5 & NA & 7 days, $4^{\circ} \mathrm{C}$ & $\begin{array}{l}\text { Mahmoud and } \\
\text { Linton, } 2008\end{array}$ \\
\hline & Salmonella enterica & & & & 5 & NA & & \\
\hline & Mesophilic & & & & NA & 2.7 & & \\
\hline & Psychrotrophic & & & & NA & 2 & & \\
\hline & Yeast and molds & & & & NA & 2.2 & & \\
\hline Spinach & Escherichia coli O157:H7 & $\mathrm{Aq}$ & 100 ppm & $5 \min$ & 2.6 & 0.13 & 7 days, $7^{\circ} \mathrm{C}$ & $\begin{array}{l}\text { Lee and Baek, } \\
2008\end{array}$ \\
\hline \multirow[t]{2}{*}{ Tomato } & Salmonella & $\mathrm{Aq}$ & 10 ppm & $5 \mathrm{~min}$ & 2.53 & 1.61 & 10 days, $4^{\circ} \mathrm{C}$ & Song et al., 2011 \\
\hline & Escherichia coli O157:H7 & & & & 2.26 & 2.23 & & \\
\hline \multirow[t]{2}{*}{ Tomato } & Alternaria alternata & $\mathrm{G}$ & 10 ppm & $1 \mathrm{~min}$ & 2.71 & Completely inactivated & 10 days, $25^{\circ} \mathrm{C}$ & Trinetta et al., 2013 \\
\hline & Stemphylium vesicarium & & & & 2.63 & Completely inactivated & & \\
\hline \multirow[t]{5}{*}{ Tomato } & Salmonella enterica & $\mathrm{G}$ & 8 ppm & $60 \mathrm{~s}$ & 2.94 & NA & 28 days, $25^{\circ} \mathrm{C}$ & Trinetta et al., 2010 \\
\hline & & & 10 ppm & $120 \mathrm{~s}$ & 3.86 & NA & & \\
\hline & & & 10 ppm & $180 \mathrm{~s}$ & 4.87 & NA & & \\
\hline & Yeast and molds & & & & & $1.57,1.47,1.54$ & & \\
\hline & Mesophilic bacteria & & & & & $1.16,2.81,3.17$ & & \\
\hline \multirow[t]{3}{*}{ Tomato } & $\begin{array}{l}\text { Listeria monocytogenes, } \\
\text { Salmonella }\end{array}$ & G & 0.5 ppm & $12 \min$ & $>5$ & NA & 28 days, $22^{\circ} \mathrm{C}$ & Bhagat et al., 2010 \\
\hline & Mesophilic & & & & NA & 0.6 & & \\
\hline & Yeast and molds & & & & NA & 0.1 & & \\
\hline \multirow[t]{2}{*}{ Tomato } & Escherichia coli & G & 3.5 ppm & 14 days & $2.9-4.7$ & 3.08 & 14 days, $20^{\circ} \mathrm{C}$ & Sun et al., 2017b \\
\hline & Alternaria alternata & & & & $1.6-4.0$ & 2.85 & & \\
\hline
\end{tabular}

Aq, aqueous; G, gaseous; NA, not available.

the shelf-life of asparagus lettuce for 10 days (Chen et al., 2010). In $\mathrm{ClO}_{2}$-treated fresh produce, including apples, green leaf lettuce, cantaloupe, and strawberries, the populations of inoculated pathogens remained relatively unchanged, whereas the growth of natural microflora was significantly delayed after 9 days of storage at $4^{\circ} \mathrm{C}$ (Rodgers et al., 2004). In lettuce, $\mathrm{ClO}_{2}$ treatment $(5.0 \mathrm{ppm}$ for $10 \mathrm{~min})$ maintained the populations of mesophilic and psychrotrophic bacteria, yeast, and mold under the detectable limit for 5 days at $4^{\circ} \mathrm{C}$ (Mahmoud and Linton, 2008). Similarly, $\mathrm{ClO}_{2}$-treated tomatoes exhibited significantly low microflora abundance during a storage period of 28 days; however, the efficacy of $\mathrm{ClO}_{2}$ varied with the exposure time and $\mathrm{ClO}_{2}$ concentration (Trinetta et al., 2010). Bhagat et al. (2010) demonstrated that treating tomato surface with 0.5 ppm $\mathrm{ClO}_{2}$ gas for 12 min delayed the growth of natural microflora and extended its shelf-life by 7 days during storage at $22^{\circ} \mathrm{C}$. Furthermore, $\mathrm{ClO}_{2}$ treatment significantly delayed the development of white molds and black spots in Roma tomato wounds inoculated with $S$. vesicarium and A. alternate (Trinetta et al., 2013). Controlled release of $\mathrm{ClO}_{2}$ (4-6 ppm) reduced the loads of E. coli, Salmonella, and A. alternata on tomatoes by 3-5 log by the end of a 14 days-storage period (Sun et al., 2017b). Moreover, strawberries packed with $\mathrm{ClO}_{2}$ generating pads exhibited reduced growth of yeast and molds until 8 days of their 12 days-storage period at $2{ }^{\circ} \mathrm{C}$ (Chiabrando et al., 2018). Similarly, $\mathrm{ClO}_{2}$ treatment reduced total aerobic bacterial and yeast and mold counts by 0.95 and $0.94 \mathrm{log}$, respectively, in grape fruit after 6 weeks of storage at $10^{\circ} \mathrm{C}$ (Sun et al., 2017a).

By contrast, $\mathrm{ClO}_{2}$ exhibits no long-term effects on reducing microbial contamination despite its initial effect. Treating cucumbers with various concentrations of $\mathrm{ClO}_{2}$, ranging from 20 to $125 \mathrm{ppm}$, did not delay mold growth during storage (Praeger et al., 2018). Although $\mathrm{ClO}_{2}$ treatment, in combination with MAP, was effective in controlling microflora on mungbean sprouts during storage, $\mathrm{ClO}_{2}$ treatment alone could not reduce the incidences of S. typhimurium and L. monocytogenes (Jin and Lee, 2007). $\mathrm{ClO}_{2}$ treatment of fresh-cut lettuce packed in MAP did not inhibit the growth of yeast during storage (López-Gálvez et al., 2010). However, 3 and 5 ppm $\mathrm{ClO}_{2}$ were more effective 
TABLE 3 | Effects of chlorine dioxide on short- and long-term reduction of microorganisms in fruits.

\begin{tabular}{|c|c|c|c|c|c|c|c|c|}
\hline \multirow[t]{2}{*}{ Produce } & \multirow[t]{2}{*}{ Microorganism } & \multicolumn{3}{|c|}{ Treatment conditions } & \multicolumn{2}{|c|}{ Log reduction } & \multirow{2}{*}{$\begin{array}{l}\text { Storage } \\
\text { conditions }\end{array}$} & \multirow[t]{2}{*}{ References } \\
\hline & & Mode & Concentration & Duration & Short-term & $\begin{array}{l}\text { Long- } \\
\text { term }\end{array}$ & & \\
\hline \multirow[t]{2}{*}{ Apples } & Escherichia coli O157:H7 & $\mathrm{Aq}$ & 3, 5 ppm & $5 \min$ & 5.6 & Unchanged & 9 days, $4^{\circ} \mathrm{C}$ & $\begin{array}{l}\text { Rodgers et al., } \\
2004\end{array}$ \\
\hline & Listeria monocytogenes & & & & 5.6 & Unchanged & & \\
\hline \multirow[t]{2}{*}{ Apples } & Salmonella & G & $4.1 \mathrm{ppm}$ & $6-25 \min$ & 4.21 & NA & 10 days, $10^{\circ} \mathrm{C}$ & Sy et al., 2005b \\
\hline & Yeasts and molds & & & & 1.68 & NA & & \\
\hline \multirow[t]{3}{*}{ Apple } & $\begin{array}{l}\text { Alicyclobacillus } \\
\text { acidoterrestris }\end{array}$ & G & 0.39 ppm & $1 \mathrm{~h}$ & 2.7 & NA & 7 days, $4^{\circ} \mathrm{C}$ & Lee et al., 2006 \\
\hline & & & 0.50 ppm & $2 \mathrm{~h}$ & 3.7 & & & \\
\hline & & & 0.60 ppm & $3 \mathrm{~h}$ & 4.5 & & & \\
\hline \multirow[t]{2}{*}{ Blueberry } & Salmonella enterica & $\mathrm{G}$ & 1.5 ppm & NA & 4.45 & 5.63 & NA & Annous et al., 2020 \\
\hline & & & 3 ppm & & 5.63 & & & \\
\hline \multirow[t]{5}{*}{ Blueberry } & Listeria monocytogenes & $\mathrm{Aq}$ & $\begin{array}{c}1,3,5,10 \\
15 \text { ppm }\end{array}$ & $\begin{array}{c}10 \mathrm{~s}, 1,5,10,20 \\
30 \mathrm{~min} ; 1,2 \mathrm{~h}\end{array}$ & $0.07-4.88$ & NA & NA & Wu and Kim, 2007 \\
\hline & Pseudomonas aeruginos & & & & $0.15-4.48$ & & & \\
\hline & Salmonella & & & & $0.12-3.32$ & & & \\
\hline & Staphylococcus aureus, & & & & $0.21-4.56$ & & & \\
\hline & Yersinia enterocolitica & & & & $0.18-3.69$ & & & \\
\hline \multirow[t]{2}{*}{ Blueberries } & Total aerobic bacteria & $\mathrm{Aq}$ & 100 ppm & $10 \mathrm{~min}$ & $1.4-1.5$ & $\begin{array}{l}1.46\left(20^{\circ}\right) \\
1.14\left(4^{\circ} \mathrm{C}\right)\end{array}$ & $\begin{array}{c}12 \text { days, } 20 \text { or } \\
4^{\circ} \mathrm{C}\end{array}$ & Chun et al., 2013 \\
\hline & Yeasts and molds & & & & $0.8-0.9$ & $1.61\left(20^{\circ}\right)$ & $0.35\left(4^{\circ} \mathrm{C}\right)$ & \\
\hline \multirow[t]{6}{*}{ Strawberry } & Escherichia coli O157:H7 & G & $\begin{array}{l}0.5,1,1.5,3 \\
5 \text { ppm }\end{array}$ & $10 \mathrm{~min}$ & 4.6 & NA & 16 days, $22^{\circ} \mathrm{C}$ & $\begin{array}{l}\text { Mahmoud et al., } \\
2007\end{array}$ \\
\hline & Listeria monocytogenes & & & & 4.7 & NA & & \\
\hline & Salmonella & & & & 4.3 & NA & & \\
\hline & Mesophilic bacteria & & & & NA & 3 & & \\
\hline & Psychrotrophic bacteria & & & & & 1.7 & & \\
\hline & Yeast and mold & & & & & 1.9 & & \\
\hline \multirow[t]{5}{*}{ Strawberry } & Escherichia coli O157:H7 & $\mathrm{Aq}$ & 5 ppm & $5 \min$ & 5.61 & Unchanged & 9 days, $4^{\circ} \mathrm{C}$ & $\begin{array}{l}\text { Rodgers et al., } \\
2004\end{array}$ \\
\hline & Listeria monocytogenes & & & & 5.61 & Unchanged & & \\
\hline & Psychrotrophic & & & & 2.5 & 2.5 & & \\
\hline & Lactic acid bacteria & & & & 1.5 & 1.7 & & \\
\hline & Yeast and mold & & & & 1.1 & 1.1 & & \\
\hline \multirow[t]{4}{*}{ Mulberry } & Mesophilic, & $\mathrm{Aq}$ & $20,60,80$ ppm & $5,10,15 \mathrm{~min}$ & $2.4-2.8$ & $2.0-2.6$ & 14 days, $-1^{\circ} \mathrm{C}$ & Chen et al., 2011 \\
\hline & Psychrotrophic & & & & $2.4-2.5$ & $2.3-2.5$ & & \\
\hline & Lactic acid bacteria & & & & $1.4-1.5$ & $1.5-1.7$ & & \\
\hline & Yeast and mold & & & & $1.0-1.1$ & $0.9-1.1$ & & \\
\hline \multirow[t]{5}{*}{ Cantaloupe } & Escherichia coli O157:H7, & G & 5.0 ppm & $5.5 \mathrm{~min}$ & 5 & NA & 12 days, $22^{\circ} \mathrm{C}$ & $\begin{array}{l}\text { Mahmoud et al., } \\
2008\end{array}$ \\
\hline & $\begin{array}{l}\text { Listeria monocytogenes, } \\
\text { Salmonella; }\end{array}$ & & & & 5 & NA & & \\
\hline & Mesophilic & & $\begin{array}{l}0.5,1,1.5,3 \\
5 \mathrm{ppm}\end{array}$ & $\begin{array}{l}0,2,4,6,8 \\
10 \min \end{array}$ & NA & 2.4 & & \\
\hline & Psychrotrophic bacteria, & & & & & 4.1 & & \\
\hline & Yeasts and molds & & & & & 2.2 & & \\
\hline Cantaloupe & $\begin{array}{c}\text { Escherichia coli O157:H7, } \\
\text { L. monocytogenes }\end{array}$ & $\mathrm{Aq}$ & 3, 5 ppm & 5 min & 5.6 & Unchanged & 9 days, $4^{\circ} \mathrm{C}$ & $\begin{array}{l}\text { Rodgers et al., } \\
2004\end{array}$ \\
\hline \multirow[t]{5}{*}{ Grape fruit } & Escherichia coli & G & 5 ppm & $24 \mathrm{~h}$ & Non-detectable & NA & $\begin{array}{c}42 \text { days, } \\
10^{\circ} \mathrm{C}+7 \text { days, } \\
20^{\circ} \mathrm{C}\end{array}$ & Sun et al., 2017a \\
\hline & Penicillium digitatum & & 60 ppm & & Non-detectable & NA & & \\
\hline & Xanthomonas citri & & $14.5,29$ ppm & & Non-detectable & NA & & \\
\hline & Total aerobic bacteria, & & & & NA & 0.95 & & \\
\hline & Yeast and mold & & & & NA & 0.94 & & \\
\hline \multirow[t]{2}{*}{ Peaches } & Salmonella, & $\mathrm{G}$ & 4.1 ppm & $6-25 \min$ & 3.23 & NA & 10 days, $10^{\circ} \mathrm{C}$ & Sy et al., 2005b \\
\hline & Yeasts and molds & & & & 2.68 & NA & & \\
\hline
\end{tabular}

Aq, aqueous; G, gaseous; NA, not available. 
TABLE 4 | Effects of chlorine dioxide on color and visual quality of fresh produce.

\begin{tabular}{|c|c|c|c|c|c|c|}
\hline Produce & Mode & $\mathrm{ClO}_{2}$ concentration & Duration & Storage & $\mathrm{ClO}_{2}$ effect & References \\
\hline \multicolumn{7}{|l|}{ Color } \\
\hline Lettuce & $\mathrm{Aq}$ & $0,5,10,20$ ppm & $10 \mathrm{~min}$ & 4 days, $4^{\circ} \mathrm{C}$ & Unaffected & Kim et al., 2008 \\
\hline Lettuce & $\mathrm{Aq}$ & 50 ppm & $10 \min$ & 8 days, $4^{\circ} \mathrm{C}$ & Unaffected & Kim et al., 2007 \\
\hline Lettuce & $\mathrm{Aq}$ & $10,40,100$ ppm & $5,10,20 \mathrm{~min}$ & 14 days, $4^{\circ} \mathrm{C}$ & Delayed degradation of color & Chen et al., 2010 \\
\hline Lettuce & G & $0.5,5.0$ ppm & $2,10 \mathrm{~min}$ & 7 days, $4^{\circ} \mathrm{C}$ & Leaf discoloration & Mahmoud and Linton, 2008 \\
\hline \multirow[t]{3}{*}{ Lettuce } & $\mathrm{G}$ & 1.4 ppm & $5.4-10.5 \min$ & 10 days, $10^{\circ} \mathrm{C}$ & Slight leaf browning & Sy et al., 2005b \\
\hline & & 2.7 ppm & 10.4-20.0 min & & Leaf browning & \\
\hline & & 4.1 ppm & 20.5-30.8 min & & Neaf browning & \\
\hline \multirow[t]{2}{*}{ Spinach } & $\mathrm{G}$ & 1-30 ppm & $20 \min$ & 7 days, $4^{\circ} \mathrm{C}$ & Unaffected & Park and Kang, 2015b \\
\hline & & 50 ppm & & & Hiher $L^{*}$ and $b^{*}$ values & \\
\hline \multirow[t]{3}{*}{ Cabbage } & G & 1.4 ppm & 5.4-10.5 min & 10 days, $10^{\circ} \mathrm{C}$ & Slight browning & Sy et al., 2005b \\
\hline & & 2.7 ppm & 10.4-20.0 min & & Leaf browning & \\
\hline & & 4.1 ppm & 20.5-30.8 min & & Leaf browning & \\
\hline \multirow[t]{2}{*}{ Tomato } & $\mathrm{Aq}$ & 10 ppm & $5 \mathrm{~min}$ & 10 days, $4^{\circ} \mathrm{C}$ & Unaffected & Song et al., 2011 \\
\hline & & 50 ppm & $20 \min$ & & Discoloration & \\
\hline Tomato & G & 8 ppm & $60 \mathrm{~s}$ & $25^{\circ} \mathrm{C}, 28$ days & Unaffected & Trinetta et al., 2010 \\
\hline Tomato & & 10 ppm & $120,180 \mathrm{~s}$ & $25^{\circ} \mathrm{C}, 28$ days & Skin wrinkling & Trinetta et al., 2010 \\
\hline Tomato & G & 0.5 ppm & $12 \min$ & 28 days, $22^{\circ} \mathrm{C}$ & Unaffected & Bhagat et al., 2010 \\
\hline \multirow[t]{3}{*}{ Tomato } & G & 1.4 ppm & $6 \min$ & 10 days, $21^{\circ} \mathrm{C}$ & Unaffected & Sy et al., 2005b \\
\hline & & 2.7 ppm & $12 \min$ & & Unaffected & \\
\hline & & $4.1 \mathrm{ppm}$ & $25 \min$ & & Unaffected & \\
\hline \multirow[t]{3}{*}{ Carrot } & G & 1.4 ppm & 5.4-10.5 min & 10 days, $10^{\circ} \mathrm{C}$ & Slight whitening in the color & Sy et al., 2005b \\
\hline & & 2.7 ppm & $10.4-20.0 \mathrm{~min}$ & & Whitening in the color & \\
\hline & & $4.1 \mathrm{ppm}$ & 20.5-30.8 min & & Whitening in the color & \\
\hline \multirow[t]{3}{*}{ Onions } & G & 1.4 ppm & $5.4 \min$ & 12 or 20 days, $21^{\circ} \mathrm{C}$ & Unaffected & Sy et al., 2005b \\
\hline & & 2.7 ppm & $10.4 \min$ & & Unaffected & \\
\hline & & 4.1 ppm & $20 \min$ & . & Unaffected & \\
\hline \multirow[t]{3}{*}{ Apple } & G & 1.4 ppm & $6 \min$ & 41 days, $21^{\circ} \mathrm{C}$ & Unaffected & \\
\hline & & 2.7 ppm & $12 \min$ & & Unaffected & \\
\hline & & 4.1 ppm & $25 \min$ & & Small brown spots on the skin & \\
\hline Cantaloupe & G & $0.5-5.0 \mathrm{mg} / \mathrm{L}$ & $0-10 \min$ & 12 days, $22^{\circ} \mathrm{C}$ & Unaffected & Mahmoud et al., 2008 \\
\hline \multirow[t]{2}{*}{ Strawberry } & G & 0.5-5 ppm & $10 \mathrm{~min}$ & 16 days, $4^{\circ} \mathrm{C}$ & Unaffected & Mahmoud et al., 2007 \\
\hline & & 29 ppm & & & Peel browning & \\
\hline \multirow[t]{2}{*}{ Strawberry } & G & NA & NA & $\begin{array}{l}3 \text { days, } 4^{\circ} \mathrm{C}+2 \text { days } \\
\text { at } 20^{\circ} \mathrm{C} / 12 \text { days, } 2^{\circ} \mathrm{C}\end{array}$ & Unaffected & Chiabrando et al., 2018 \\
\hline & & & & 12 days, $2^{\circ} \mathrm{C}$ & Unaffected & \\
\hline \multirow[t]{2}{*}{ Strawberry } & $\mathrm{Aq}$ & 5 ppm & NA & 3 weeks, $4^{\circ} \mathrm{C}$ & Maintained $L$ and a values & Aday and Caner, 2011 \\
\hline & & & & & Decreased $L$ and a values & \\
\hline \multirow[t]{3}{*}{ Peaches } & G & 1.4 ppm & $5.4 \min$ & 10 days, $21^{\circ} \mathrm{C}$ & Browning & Sy et al., 2005b \\
\hline & & 2.7 ppm & $10.4 \min$ & . & Browning & \\
\hline & & 4.1 ppm & $20 \min$ & . & Browning & \\
\hline \multicolumn{7}{|c|}{ Visual quality } \\
\hline Tomato & $\mathrm{G}$ & 5 ppm & $12 \mathrm{~h}$ & 20 days, $5^{\circ} \mathrm{C}$ & Delayed color development & Islam et al., 2017 \\
\hline \multirow[t]{3}{*}{ Apple } & $\mathrm{G}$ & 0.39-0.60 ppm & $1-3 h$ & 7 days, $4^{\circ} \mathrm{C}$ & Unaffected & Lee et al., 2006 \\
\hline & & $1.78-2.69$ ppm & $1-3 h$ & & Black spots on the fruit surface & \\
\hline & & 4.32-6.55 ppm & $1-3 h$ & & Black spots on the fruit surface & \\
\hline \multirow[t]{2}{*}{ Grapefruit } & G & 14.5 ppm & 10 days & $\begin{array}{c}42 \text { days, } \\
10^{\circ} \mathrm{C}+7 \text { days, } 20^{\circ} \mathrm{C}\end{array}$ & Maintained & Sun et al., 2017a \\
\hline & & 29 ppm & & & Peel browning & \\
\hline
\end{tabular}

Aq, aqueous; G, gaseous; NA, not available. 
against L. monocytogenes than yeasts and molds during cold storage (Rodgers et al., 2004).

\section{EFFECTS OF CHLORINE DIOXIDE TREATMENT ON THE POSTHARVEST QUALITY OF FRESH PRODUCE}

\section{Color}

Color is one of the fundamental characteristics that determines the visual quality and acceptability of fresh produce. Depending on the concentration, $\mathrm{ClO}_{2}$ differentially affects the appearance of treated fresh produce (Table 4). However, previous studies suggest that $\mathrm{ClO}_{2}$ has no effect on the color of fresh produce; $\mathrm{ClO}_{2}$ exposure had no effect on Hunter $\mathrm{L}$, $\mathrm{a}$, and $\mathrm{b}$ values of tomatoes, spinach, and lettuce (Kim et al., 2007; Song et al., 2011; Hassenberg et al., 2014; Park and Kang, 2015a). Similarly, treatment with $0.5 \mathrm{ppm} \mathrm{ClO}_{2}$ gas for $12 \mathrm{~min}$ did not significantly affect the color of orange peel (Bhagat et al., 2011). Furthermore, the appearance of blueberries was not affected by long-term $\mathrm{ClO}_{2}$ exposure (2-12 h) (Popa et al., 2007; Wu and Kim, 2007). By contrast, higher concentrations of $\mathrm{ClO}_{2}$ result in the bleaching of fresh produce. For example, strawberries treated with $\mathrm{ClO}_{2}$ underwent white bleaching after 8 days of storage at $2^{\circ} \mathrm{C}$ (Chiabrando et al., 2018). Oxidation of oligosaccharides, such as cellulose and hemicellulose, and chlorophyll, has been hypothesized as the possible cause of bleaching in fresh produce (Singh et al., 2002; Chen and Zhu, 2011).

Chlorine dioxide has been reported to differentially affect enzymatic browning of fresh produce, resulting from the oxidation of phenols to o-quinones that is catalyzed by polyphenol oxidase (PPO; Altunkaya and Gökmen, 2009) during postharvest handling and processing. In grapes, repeated application of $\mathrm{ClO}_{2}$ during storage significantly decreased rachis browning (Chen et al., 2018). Reduced browning in a variety of fresh produce, such as fresh-cut asparagus lettuce, and apples, is associated with decreased PPO activity (Fu et al., 2007; Chen et al., 2010). This can be attributed to the oxidation of disulfide bonds and amino acids at the active site of PPO by $\mathrm{ClO}_{2}$ (Fu et al., 2007). By contrast, $\mathrm{ClO}_{2}$ treatment may also cause browning of fresh produce. For example, $\mathrm{ClO}_{2}$ treatment resulted in rapid color change in spinach leaves, browning of grapefruit, cabbage, lettuce, peaches, and apples (Sy et al., 2005b; Lee et al., 2006; Mahmoud and Linton, 2008; Park and Kang, 2015a; Sun et al., 2017a).

\section{Firmness}

Firmness, another important quality-determining characteristic, influences consumer appeal and the commercial value of fresh produce. Effect of $\mathrm{ClO}_{2}$ treatment on firmness and weight loss of fresh produce is summarized in Table 5. $\mathrm{ClO}_{2}$ treatment retains the firmness of several fresh fruits, such as strawberries, plums, apricots, and mangoes, during postharvest storage (Aday and Caner, 2011; Chen and Zhu, 2011; Zhang et al., 2019). Furthermore, controlled-release of $\mathrm{ClO}_{2}$ gas has been reported to regulate the firmness of non-inoculated and E. coli- and C. acutatum-inoculated berries during storage (Sun et al., 2014).

After harvesting, respiration and transpiration continue in fresh produce, and carbohydrate and water reserves are continually consumed without replacement, leading to progressive loss of turgidity and weight during storage. Fruit moisture and weight loss are associated with decreased fruit firmness, shrinking, and shriveling (Paniagua et al., 2013; Lufu et al., 2020). However, $\mathrm{ClO}_{2}$ reduces the rate of water loss in the $\mathrm{ClO}_{2}$-treated produce (Guo et al., 2014; Wang et al., 2014). The application of $\mathrm{ClO}_{2}$ at low concentrations for long durations in active packaging material has been shown to improve fruit firmness and reduce water loss (Guo et al., 2014).

Less weight loss in $\mathrm{ClO}_{2}$-treated berries is associated with $50 \%$ closed stomata during storage at low temperatures (Wang et al., 2014). In general, fruit ripening is associated with a climacteric increase in ethylene production and extensive modifications in cell wall polysaccharides. $\mathrm{ClO}_{2}$ delays the increase in respiration rate and ethylene biosynthesis, resulting in delayed ripening that further leads to delayed fruit softening (Chen and Zhu, 2011; Guo et al., 2013, 2014). $\mathrm{ClO}_{2}$ may also alter tissue metabolism by oxidizing cell constituents, thereby leading to changes in respiration, and, in turn, inhibiting weight loss and maintaining fruit firmness (Gomez-Lopez et al., 2008).

\section{Sensory Properties}

Previous studies have reported that $\mathrm{ClO}_{2}$ treatment can retain the sensory properties of fresh produce. Effect of $\mathrm{ClO}_{2}$ treatment on sensory properties of fresh produce is summarized in Table 6. For example, gaseous $\mathrm{ClO}_{2}$ treatment $(4.1 \mathrm{ppm})$ did not compromise

TABLE 5 | Effects of chlorine dioxide on firmness and weight loss of fresh produce.

\begin{tabular}{|c|c|c|c|c|c|c|c|}
\hline Produce & Mode & $\mathrm{ClO}_{2}$ concentration & Duration & Weight loss & Firmness & Storage & References \\
\hline Spinach & G & $1-30 \mathrm{ppm}$ & $20 \mathrm{~min}$ & & Unaffected & 7 days, $4^{\circ} \mathrm{C}$ & Park and Kang, 2015b \\
\hline Spinach & G & $1-50 \mathrm{ppm}$ & $20 \mathrm{~min}$ & & Unaffected & 7 days, $4^{\circ} \mathrm{C}$ & Park and Kang, 2015b \\
\hline Grape tomatoes & G & 2-3.5 ppm & 14 days & Reduced & Increased & 14 days, $20^{\circ} \mathrm{C}$ & Sun et al., 2017b \\
\hline Grape tomatoes & G & $2,4,6,8 \mathrm{ppm}$ & 14 days & Reduced & Increased & 14 days, $20^{\circ} \mathrm{C}$ & Sun et al., 2017c \\
\hline Cherry tomatoes & G & $2,4,6,8 \mathrm{ppm}$ & 14 days & Reduced & Maintained & 14 days, $20^{\circ} \mathrm{C}$ & Sun et al., 2017c \\
\hline Tomato & G & 10 ppm & $120,180 \mathrm{~s}$ & & Skin wrinkling & $25^{\circ} \mathrm{C}, 28$ days & Trinetta et al., 2010 \\
\hline Tomato & G & 5 ppm & $12 \mathrm{~h}$ & Reduced & Increased & 20 days, $5^{\circ} \mathrm{C}$ & Islam et al., 2017 \\
\hline \multirow[t]{2}{*}{ Strawberry } & $\mathrm{Aq}$ & $5 \mathrm{ppm}$ & NA & & Reduced & 3 weeks, $4^{\circ} \mathrm{C}$ & Aday and Caner, 2011 \\
\hline & & 10 ppm & & & Increased & & \\
\hline Blueberry & $G$ & $1-2.5 \mathrm{ppm}$ & 9 days & & Maintained & 9 days, $10^{\circ} \mathrm{C}$ & Sun et al., 2014 \\
\hline
\end{tabular}

Aq, aqueous; G, gaseous; NA, not available. 
TABLE 6 | Effects of chlorine dioxide on sensory properties of fresh produce.

\begin{tabular}{|c|c|c|c|c|c|c|}
\hline Produce & Mode & $\mathrm{ClO}_{2}$ concentration & Duration & Storage & Sensory property & References \\
\hline Lettuce & $\mathrm{Aq}$ & 3 ppm & $1 \mathrm{~min}$ & 3 days, $4^{\circ} \mathrm{C}+7$ days, $8^{\circ} \mathrm{C}$ & Unaffected & López-Gálvez et al., 2010 \\
\hline Lettuce & $\mathrm{Aq}$ & 50 ppm & $10 \mathrm{~min}$ & 8 days, $4^{\circ} \mathrm{C}$ & Unaffected & Kim et al., 2007 \\
\hline Lettuce & $\mathrm{Aq}$ & $3,5 \mathrm{ppm}$ & & $48 \mathrm{~h}, 4^{\circ} \mathrm{C}$ & Unaffected & Rodgers et al., 2004 \\
\hline Lettuce & $\mathrm{Aq}$ & $10,40,100 \mathrm{ppm}$ & $5,10,20 \mathrm{~min}$ & 14 days, $4^{\circ} \mathrm{C}$ & Unaffected & Chen et al., 2010 \\
\hline Lettuce & $\mathrm{G}$ & 1.4 ppm & $5.4-10.5 \mathrm{~min}$ & 10 days, $10^{\circ} \mathrm{C}$ & Decreased & Sy et al., 2005b \\
\hline Cabbage & G & 1.4 ppm & $5.4-10.5 \mathrm{~min}$ & 10 days, $10^{\circ} \mathrm{C}$ & Decreased & Sy et al., 2005b \\
\hline Carrot & $G$ & 1.4 ppm & $5.4-10.5 \mathrm{~min}$ & 10 days, $10^{\circ} \mathrm{C}$ & Decreased & Sy et al., 2005b \\
\hline Tomato & $G$ & $1.4 \mathrm{ppm}$ & $6 \mathrm{~min}$ & 10 days, $21^{\circ} \mathrm{C}$ & Unaffected & Sy et al., 2005b \\
\hline Onions & G & $1.4 \mathrm{ppm}$ & $5.4 \mathrm{~min}$ & 12 or 20 days, $21^{\circ} \mathrm{C}$ & Unaffected & Sy et al., 2005b \\
\hline Apple & $\mathrm{Aq}$ & 3, 5 ppm & & $48 \mathrm{~h}, 4^{\circ} \mathrm{C}$ & Unaffected & Rodgers et al., 2004 \\
\hline Strawberry & $\mathrm{Aq}$ & 3, 5 ppm & & $48 \mathrm{~h}, 4^{\circ} \mathrm{C}$ & Unaffected & Rodgers et al., 2004 \\
\hline \multirow[t]{2}{*}{ Strawberry } & $\mathrm{Aq}$ & 5 ppm & & 3 weeks, $4^{\circ} \mathrm{C}$ & Maintained & Aday and Caner, 2011 \\
\hline & & & & & Maintained & \\
\hline Cantaloupe & $\mathrm{Aq}$ & 3, 5 ppm & & $48 \mathrm{~h}, 4^{\circ} \mathrm{C}$ & Unaffected & Rodgers et al., 2004 \\
\hline Blueberry & G & $4 \mathrm{ppm}$ & $12 \mathrm{~h}$ & Overnight, $4^{\circ} \mathrm{C}$ & Improved & Popa et al., 2007 \\
\hline Apple & G & 1.4 ppm & $6 \mathrm{~min}$ & & Unaffected & \\
\hline \multirow[t]{3}{*}{ Peaches } & G & 1.4 ppm & $5.4 \mathrm{~min}$ & 10 days, $21^{\circ} \mathrm{C}$ & Decreased & Sy et al., 2005b \\
\hline & & 2.7 ppm & $10.4 \mathrm{~min}$ & & Decreased & \\
\hline & & $4.1 \mathrm{ppm}$ & $20 \mathrm{~min}$ & & Decreased & \\
\hline Grapefruit & G & $14.5 \mathrm{ppm}$ & 10 days & 42 days, $10^{\circ} \mathrm{C}+7$ days, $20^{\circ} \mathrm{C}$ & Maintained & Sun et al., 2017a \\
\hline
\end{tabular}

Aq, aqueous; G, gaseous.

the sensory qualities of blueberries, strawberries, and raspberries stored for 10 days at $8^{\circ} \mathrm{C}$ (Sy et al., 2005a). Similar results were obtained for fresh-cut cabbage, carrot, and iceberg lettuce treated with 3-5 ppm $\mathrm{ClO}_{2}$ (Rodgers et al., 2004; Sy et al., 2005b; LópezGálvez et al., 2010). Moreover, $\mathrm{ClO}_{2}$ treatment positively affects the composition of volatile compounds and free amino acids in citrus fruits, resulting in the retention of their distinct flavor (Liu et al., 2020). Furthermore, $\mathrm{ClO}_{2}$-treated plums maintain high sensory properties during storage (Chen and Zhu, 2011). Few studies revealed that the sensory properties of fresh produce can be improved by $\mathrm{ClO}_{2}$ application. For instance, $\mathrm{ClO}_{2}$-treated strawberries, blueberries, and mulberries exhibited better sensory scores than the untreated controls (Jin and Lee, 2007; Wu and Kim, 2007; Chen et al., 2011; Chun et al., 2013). Nonetheless, 20 ppm $\mathrm{ClO}_{2}$ significantly affected the sensory properties of lettuce and cabbage (Gomez-Lopez et al., 2008).

\section{CHLORINE DIOXIDE APPLICATION: EFFICACY AND LIMITATIONS}

Because of its high oxidative capacity (2.5-fold that of chlorine), $\mathrm{ClO}_{2}$ is effective in microbial inactivation at concentration as low as $0.1 \mathrm{ppm}$ with minimal contact time (Praeger et al., 2018). Most importantly, $\mathrm{ClO}_{2}$ is effective against both Gram-positive and Gram-negative bacteria, whereas molds and yeasts showed intermediate tolerance (Yoon and Lee, 2018; Sun et al., 2019). Additionally, $\mathrm{ClO}_{2}$ does not react with organic matter to form carcinogenic by-products such as trihalomethanes which makes $\mathrm{ClO}_{2}$ to be effective over a wide $\mathrm{pH}$ range (Praeger et al., 2018). In the United States, a maximum $3 \mathrm{ppm}$ of $\mathrm{ClO}_{2}$ is allowed for fresh produce treatment. In Europe, rinsing with potable water is necessary following the $\mathrm{ClO}_{2}$ treatment (Praeger et al., 2018).

Comparison of disinfection efficacy of various sanitizers revealed that gaseous $\mathrm{ClO}_{2}$, hydrostatic pressure and electrolyzed oxidizing water were more effective in microbial inactivation than other sanitizers. The average microbial reductions of $\mathrm{ClO}_{2}$ gas, hydrostatic pressure and electrolyzed oxidizing water were 4.07, 3.94, and $3.01 \mathrm{log}$, respectively (Yoon and Lee, 2018). On the other hand, the average microbial inactivation of aqueous $\mathrm{ClO}_{2}$ (1.49 log) was less than gaseous $\mathrm{ClO}_{2}$, however, it was still higher than chlorine-based disinfectants (1.12 log) (Yoon and Lee, 2018). Higher antimicrobial activity of gaseous $\mathrm{ClO}_{2}$ may attribute to its easier accessibility to microbes located in the unreachable parts the fresh produce. Moreover, $\mathrm{ClO}_{2}$ gas can readily diffuse into the tissues of fresh produce, hence, it may inactivate internalized microbes (Yoon and Lee, 2018). However, handling with gaseous $\mathrm{ClO}_{2}$ is inconvenient as it needs to be produced onsite. Moreover, it is expensive and requires technical expertise.

The major limitations of $\mathrm{ClO}_{2}$ for practical applications include it may not be effective at permitted concentrations; it may affect quality of treated fresh produce in some instances. Since $\mathrm{ClO}_{2}$ is highly explosive and toxic to humans at higher concentrations, it is challenging to implement this treatment technology at industry scale.

\section{CONCLUSION AND FUTURE PERSPECTIVES}

Chlorine dioxide application, in gaseous and aqueous forms, has been demonstrated to be effective in controlling microbial growth and retaining the quality of fresh produce, however, it is largely depending upon the respective produce type and treatment conditions. Gaseous $\mathrm{ClO}_{2}$ is more effective than the aqueous form. Nevertheless, although aqueous $\mathrm{ClO}_{2}$ solutions may be easy to use, they require an additional washing step. $\mathrm{ClO}_{2}$, whether in gaseous or aqueous form, destabilizes cell membranes, alters membrane permeability, and interrupts 
protein synthesis in microbes, along with influencing ethylene biosynthesis and respiration rate in fresh produce, which are crucial for maintaining the quality of fresh produce. In general, initial reduction in microbial load significantly affects microbial contamination during storage of fresh produce, thereby resulting in an extended shelf-life. Previous studies suggest that $\mathrm{ClO}_{2}$ concentration and exposure time are crucial in determining the efficacy of $\mathrm{ClO}_{2}$ against microbes, but a holistic approach is required to unravel the mechanisms underlying the regulation of fresh produce quality by $\mathrm{ClO}_{2}$.

Our review showed that current research on disinfection by $\mathrm{ClO}_{2}$ has mainly focused on the bactericidal effects of $\mathrm{ClO}_{2}$; recently, studies on antifungal and antiviral effects of $\mathrm{ClO}_{2}$, are gaining attention. Currently, the efficacy of $\mathrm{ClO}_{2}$ has been mostly tested at the laboratory level, thus, highlighting the need for industrial-level testing for various types of fresh produce. Disposition and chemical fate of $\mathrm{ClO}_{2}$ gas on treated fresh produce are not well understood; therefore, further studies should focus on this dimension, which has been largely neglected

\section{REFERENCES}

Aday, M. S., and Caner, C. (2011). The Applications of 'active packaging and chlorine dioxide' for extended shelf life of fresh strawberries. Packaging Technol. Sci. 24, 123-136. doi: 10.1002/pts.918

Altunkaya, A., and Gökmen, V. (2009). Effect of various anti-browning agents on phenolic compounds profile of fresh lettuce (L. sativa). Food Chem. 117, 122-126. doi: 10.1016/j.foodchem.2009.03.085

Annous, B. A., Buckley, D., and Burke, A. (2020). Evaluation of Chlorine Dioxide Gas against Four Salmonella enterica Serovars Artificially Contaminated on Whole Blueberries. J. Food Prot. 83, 412-417. doi: 10.4315/0362-028X.JFP19-452

Behlau, F., Paloschi, A., Marin, T. G. S., Santos, T. A., Ferreira, H., and Nascimento, L. M. D. (2021). Chlorine dioxide, peroxyacetic acid, and calcium oxychloride for post-harvest decontamination of citrus fruit against Xanthomonas citri subsp. citri, causal agent of citrus canker. Crop Protect. 146:105679. doi: 10. 1016/j.cropro.2021.105679

Berg, J. D., Roberts, P. V., and Matin, A. (1986). Effect of chlorine dioxide on selected membrane functions of Escherichia coli. J. Appl. Bacteriol. 60, 213-220. doi: 10.1111/j.1365-2672.1986.tb01075.x

Bhagat, A., Mahmoud, B. S., and Linton, R. H. (2010). Inactivation of Salmonella enterica and Listeria monocytogenes inoculated on hydroponic tomatoes using chlorine dioxide gas. Foodborne Pathog. Dis. 7, 677-685. doi: 10.1089/fpd.2009. 0466

Bhagat, A., Mahmoud, B. S., and Linton, R. H. (2011). Effect of chlorine dioxide gas on Salmonella enterica inoculated on navel orange surfaces and its impact on the quality attributes of treated oranges. Foodborne Pathog. Dis. 8, 77-85. doi: 10.1089/fpd.2010.0622

Bridges, D. F., Lacombe, A., and Wu, V. C. H. (2020). Integrity of the Escherichia coli O157:H7 Cell Wall and Membranes After Chlorine Dioxide Treatment. Front. Microbiol. 11:888. doi: 10.3389/fmicb.2020.00888

Buzby, J. C., Farah-Wells, H., and Hyman, J. (2014). The Estimated Amount, Value, and Calories of Postharvest Food Losses at the Retail and Consumer Levels in the United States. USDA ERS Econom. Informat. Bull. Number 121:2501659. doi: $10.2139 /$ ssrn.2501659

CDC (2018). National Outbreak Reporting System (NORS). Atlanta, GA: CDC.

Chacha, J. S., Zhang, L., Ofoedu, C. E., Suleiman, R. A., Dotto, J. M., Roobab, U., et al. (2021). Revisiting Non-Thermal Food Processing and Preservation Methods-Action Mechanisms, Pros and Cons: A Technological Update (20162021). Foods 10:foods10061430. doi: 10.3390/foods 10061430

Chen, S., Wang, H., Wang, R., Fu, Q., and Zhang, W. (2018). Effect of gaseous chlorine dioxide $\left(\mathrm{ClO}_{2}\right)$ with different concentrations and numbers in studies on $\mathrm{ClO}_{2}$ disinfection. Moreover, this review did not assess the different methods of $\mathrm{ClO}_{2}$ generation and the efficacy of $\mathrm{ClO}_{2}$ in combination with other technologies for postharvest quality and microbial safety of fresh produce.

\section{AUTHOR CONTRIBUTIONS}

M-HP: supervision. SM and M-HP: conceptualization, writingoriginal draft preparation, contributed to the article, and approved the submitted version.

\section{FUNDING}

This study was funded by the Cooperative Research Program for Agriculture, Science, and Technology (Project No. PJ01502903) in the Rural Development Administration of the Republic of Korea.

of treatments on controlling berry decay and rachis browning of table grape J. Food Proces. Preservat. 42:13662. doi: 10.1111/jfpp.13662

Chen, Z., and Zhu, C. (2011). Combined effects of aqueous chlorine dioxide and ultrasonic treatments on postharvest storage quality of plum fruit (Prunus salicina L.). Postharv. Biol. Technol. 61, 117-123. doi: 10.1016/j.postharvbio. 2011.03.006

Chen, Z., Zhu, C., and Han, Z. (2011). Effects of aqueous chlorine dioxide treatment on nutritional components and shelf-life of mulberry fruit (Morus alba L.). J. Biosci. Bioeng. 111, 675-681. doi: 10.1016/j.jbiosc.2011.01.010

Chen, Z., Zhu, C., Zhang, Y., Niu, D., and Du, J. (2010). Effects of aqueous chlorine dioxide treatment on enzymatic browning and shelf-life of fresh-cut asparagus lettuce (Lactuca sativa L.). Postharv. Biol. Technol. 58, 232-238. doi: 10.1016/j. postharvbio.2010.06.004

Chiabrando, V., Giuggioli, N., Maghenzani, M., Peano, C., and Giacalone, G. (2018). Improving Storability of Strawberries with Gaseous Chlorine Dioxide in Perforated Clamshell Packaging. Polish J. Food Nutrit. Sci. 68, 141-148. doi: 10.1515/pjfns-2017-0024

Chumyam, A., Shank, L., Uthaibutra, J., and Saengnil, K. (2016). Effects of chlorine dioxide on mitochondrial energy levels and redox status of 'Daw' longan pericarp during storage. Postharv. Biol. Technol. 116, 26-35. doi: 10.1016/j. postharvbio.2016.01.002

Chun, H. H., Yu, D. J., and Song, K. B. (2013). Effects of combined nonthermal treatment on microbial growth and the quality of minimally processed yam (Dioscorea japonicaThunb) during storage. Int. J. Food Sci. Technol. 48, 334340. doi: 10.1111/j.1365-2621.2012.03191.x

Deng, L. Z., Mujumdar, A. S., Pan, Z., Vidyarthi, S. K., Xu, J., Zielinska, M., et al. (2020). Emerging chemical and physical disinfection technologies of fruits and vegetables: a comprehensive review. Crit. Rev. Food Sci. Nutr. 60, 2481-2508. doi: 10.1080/10408398.2019.1649633

Du, J., Han, Y., and Linton, R. H. (2002). Inactivation by chlorine dioxide gas $\left(\mathrm{ClO}_{2}\right)$ of Listeria monocytogenes spotted onto different apple surfaces. Food Microbiol. 19, 481-490. doi: 10.1006/fmic.2002.0501

Du, J., Han, Y., and Linton, R. H. (2003). Efficacy of chlorine dioxide gas in reducing Escherichia coli O157:H7 on apple surfaces. Food Microbiol. 20, 583591. doi: 10.1016/s0740-0020(02)00129-6

EPA (1999). Alternative disinfectants and oxidants, guidance manual. Washington, D.C: Environmental Protection Agency.

European Food Safety Authority (2021). The European Union One Health 2019 Zoonoses Report. EFSA J. 19:e06406. doi: 10.2903/j.efsa.2021.6406

FAO (2011). Global food losses and food waste. Rome: FAO.

FDA (2008). Guidance for industry: guide to minimize microbial food safety hazards for fresh fruits and vegetables. Silver Spring: FDA. 
Fu, Y., Zhang, K., Wang, N., and Du, J. (2007). Effects of aqueous chlorine dioxide treatment on polyphenol oxidases from Golden Delicious apple. LWT Food Sci. Technol. 40, 1362-1368. doi: 10.1016/j.lwt.2006.11.001

Ge, Y., Zhang, X., Shu, L., and Yang, X. (2021). Kinetics and Mechanisms of Virus Inactivation by Chlorine Dioxide in Water Treatment: A Review. Bull. Environ. Contam. Toxicol. 106, 560-567. doi: 10.1007/s00128-021-03137-3

Gomez-Lopez, V. M., Devlieghere, F., Ragaert, P., and Debevere, J. (2007). Shelflife extension of minimally processed carrots by gaseous chlorine dioxide. Int. J. Food Microbiol. 116, 221-227. doi: 10.1016/j.ijfoodmicro.2006.12.008

Gomez-Lopez, V. M., Ragaert, P., Jeyachchandran, V., Debevere, J., and Devlieghere, F. (2008). Shelf-life of minimally processed lettuce and cabbage treated with gaseous chlorine dioxide and cysteine. Int. J. Food Microbiol. 121, 74-83. doi: 10.1016/j.ijfoodmicro.2007.11.036

Guo, Q., Lv, X., Xu, F., Zhang, Y., Wang, J., Lin, H., et al. (2013). Chlorine dioxide treatment decreases respiration and ethylene synthesis in fresh-cut 'Hami' melon fruit. Int. J. Food Sci. Technol. 48, 1775-1782. doi: 10.1111/ijfs.12149

Guo, Q., Wu, B., Peng, X., Wang, J., Li, Q., Jin, J., et al. (2014). Effects of chlorine dioxide treatment on respiration rate and ethylene synthesis of postharvest tomato fruit. Postharv. Biol. Technol. 93, 9-14. doi: 10.1016/j.postharvbio.2014. 01.013

Han, Y., Floros, J. D., Linton, R. H., Nielsen, S. S., and Nelson, P. E. (2001a). Response surface modeling for the inactivation of Escherichia coli O157:H7 on green peppers (Capsicum annuum L.) by chlorine dioxide gas treatments. J. Food Prot. 64, 1128-1133. doi: 10.4315/0362-028x-64.8.1128

Han, Y., Linton, R. H., Nielsen, S. S., and Nelson, P. E. (2001b). Reduction of Listeria monocytogenes on green peppers (Capsicum annuum L.) by gaseous and aqueous chlorine dioxide and water washing and its growth at 7 degrees C. J. Food Prot. 64, 1730-1738. doi: 10.4315/0362-028x-64.11.1730

Han, Y., Sherman, D. M., Linton, R. H., Nielsen, S. S., and Nelson, P. E. (2000). The effects of washing and chlorine dioxide gas on survival and attachment of Escherichia coli O157: H7 to green pepper surfaces. Food Microbiol. 17, 521-533. doi: $10.1006 /$ fmic. 2000.0343

Hassenberg, K., Herppich, W. B., and Praeger, U. (2014). Chlorine dioxide for the reduction of human pathogens in lettuce washing process. Landtechnik 69, $185-189$.

Islam, M. Z., Mahmuda, A. M., Jong, M. P., Il-Seop, K., and Ho-Min, K. (2017). Chlorine dioxide gas retain postharvest quality and shelf life of tomato during modified atmosphere packaging storage. AGRIVITA J. Agricult. Sci. 39. doi: 10.17503/agrivita.v39i3.1454

Jin, H. H., and Lee, S. Y. (2007). Combined effect of aqueous chlorine dioxide and modified atmosphere packaging on inhibiting Salmonella typhimurium and Listeria monocytogenes in mungbean sprouts. J. Food Sc.i 72, M441-M445. doi: 10.1111/j.1750-3841.2007.00555.x

Jin, M., Shan, J., Chen, Z., Guo, X., Shen, Z., Qiu, Z., et al. (2013). Chlorine dioxide inactivation of enterovirus 71 in water and its impact on genomic targets. Environ. Sci. Technol. 47, 4590-4597. doi: 10.1021/es305282g

Joshi, K., Mahendran, R., Alagusundaram, K., Norton, T., and Tiwari, B. K. (2013). Novel disinfectants for fresh produce. Trends Food Sci. Technol. 34, 54-61. doi: 10.1016/j.tifs.2013.08.008

Keskinen, L. A., Burke, A., and Annous, B. A. (2009). Efficacy of chlorine, acidic electrolyzed water and aqueous chlorine dioxide solutions to decontaminate Escherichia coli O157:H7 from lettuce leaves. Int. J. Food Microbiol. 132, 134140. doi: 10.1016/j.ijfoodmicro.2009.04.006

Kim, S., Lee, H., Ryu, J. H., and Kim, H. (2017). Inactivation of Bacillus cereus Spores on Red Chili Peppers Using a Combined Treatment of Aqueous Chlorine Dioxide and Hot-Air Drying. J. Food Sci. 82, 1892-1897. doi: 10.1111/ 1750-3841.13771

Kim, Y. J., Lee, S. H., and Song, K. B. (2007). Effect of aqueous chlorine dioxide treatment on the microbial growth and qualities of iceberg lettuce during storage. J. Appl. Biol. Chem. 50, 239-243.

Kim, Y. J., Lee, S. H., Park, J., Park, J., Chung, M., Kwon, K., et al. (2008). Inactivation of Escherichia coli O157:H7, Salmonella typhimurium, and Listeria monocytogenes on stored iceberg lettuce by aqueous chlorine dioxide treatment. J. Food Sci. 73, M418-M422. doi: 10.1111/j.1750-3841.2008.00940.x

Kingsley, D. H., and Annous, B. A. (2019). Evaluation of Steady-State Gaseous Chlorine Dioxide Treatment for the Inactivation of Tulane virus on Berry Fruits. Food Environ. Virol. 11, 214-219. doi: 10.1007/s12560-019-09 $382-4$
Kingsley, D. H., Perez-Perez, R. E., Niemira, B. A., and Fan, X. (2018). Evaluation of gaseous chlorine dioxide for the inactivation of Tulane virus on blueberries. Int. J. Food Microbiol. 273, 28-32. doi: 10.1016/j.ijfoodmicro.2018.01.024

Lee, H., Beuchat, L. R., Ryu, J. H., and Kim, H. (2018). Inactivation of Salmonella typhimurium on red chili peppers by treatment with gaseous chlorine dioxide followed by drying. Food Microbiol. 76, 78-82. doi: 10.1016/j.fm.2018.04.016

Lee, S. Y., and Baek, S. Y. (2008). Effect of chemical sanitizer combined with modified atmosphere packaging on inhibiting Escherichia coli O157:H7 in commercial spinach. Food Microbiol. 25, 582-587. doi: 10.1016/j.fm.2008. 02.003

Lee, S. Y., Dancer, G. I., Chang, S. S., Rhee, M. S., and Kang, D. H. (2006). Efficacy of chlorine dioxide gas against Alicyclobacillus acidoterrestris spores on apple surfaces. Int. J. Food Microbiol. 108, 364-368. doi: 10.1016/j.ijfoodmicro.2005. 11.023

Li, J. W., Xin, Z. T., Wang, X. W., Zheng, J. L., and Chao, F. H. (2004). Mechanisms of inactivation of hepatitis A virus in water by chlorine dioxide. Water Res. 38, 1514-1519. doi: 10.1016/j.watres.2003.12.021

Lin, X., Chen, G., Jin, T. Z., Wen, M., Wu, J., Wen, J., et al. (2021). Extension of shelf life of semi-dry longan pulp with gaseous chlorine dioxide generating film. Int. J. Food Microbiol. 337:108938. doi: 10.1016/j.ijfoodmicro.2020.108938

Liu, X., Jiao, W., Du, Y., Chen, Q., Su, Z., and Fu, M. (2020). Chlorine Dioxide Controls Green Mold Caused by Penicillium digitatum in Citrus Fruits and the Mechanism Involved. J. Agric. Food Chem. 68, 13897-13905. doi: 10.1021/acs. jafc.0c05288

López-Gálvez, F., Allende, A., Truchado, P., Martínez-Sánchez, A., Tudela, J. A., Selma, M. V., et al. (2010). Suitability of aqueous chlorine dioxide versus sodium hypochlorite as an effective sanitizer for preserving quality of fresh-cut lettuce while avoiding by-product formation. Postharv. Biol. Technol. 55, 53-60. doi: 10.1016/j.postharvbio.2009.08.001

Lufu, R., Ambaw, A., and Opara, U. L. (2020). Water loss of fresh fruit: Influencing pre-harvest, harvest and postharvest factors. Sci. Horticult. 272:109519. doi: 10.1016/j.scienta.2020.109519

Mahmoud, B. S., and Linton, R. H. (2008). Inactivation kinetics of inoculated Escherichia coli O157:H7 and Salmonella enterica on lettuce by chlorine dioxide gas. Food Microbiol. 25, 244-252. doi: 10.1016/j.fm.2007.10.015

Mahmoud, B. S., Bhagat, A. R., and Linton, R. H. (2007). Inactivation kinetics of inoculated Escherichia coli O157:H7, Listeria monocytogenes and Salmonella enterica on strawberries by chlorine dioxide gas. Food Microbiol. 24, 736-744. doi: $10.1016 /$ j.fm.2007.03.006

Mahmoud, B. S., Vaidya, N. A., Corvalan, C. M., and Linton, R. H. (2008). Inactivation kinetics of inoculated Escherichia Coli O157:H7, Listeria monocytogenes and Salmonella Poona on whole cantaloupe by chlorine dioxide gas. Food Microbiol. 25, 857-865. doi: 10.1016/j.fm.2008.05.009

Mahovic, M. J., Tenney, J. D., and Bartz, J. A. (2007). Applications of Chlorine Dioxide Gas for Control of Bacterial Soft Rot in Tomatoes. Plant Dis. 91, 1316-1320. doi: 10.1094/PDIS-91-10-1316

Netramai, S., Kijchavengkul, T., Sakulchuthathip, V., and Rubino, M. (2016). Antimicrobial efficacy of gaseous chlorine dioxide against Salmonella enterica Typhimurium on grape tomato (Lycopersicon esculentum). Int. J. Food Sci. Technol. 51, 2225-2232. doi: 10.1111/ijfs.13209

Ofori, I., Maddila, S., Lin, J., and Jonnalagadda, S. B. (2018). Chlorine dioxide inactivation of Pseudomonas aeruginosa and Staphylococcus aureus in water: The kinetics and mechanism. J. Water Process Engine. 26, 46-54. doi: 10.1016/j. jwpe.2018.09.001

Olanya, O. M., Annous, B. A., and Taylor, J. (2015). Effects of Pseudomonas chlororaphis and gaseous chlorine dioxide on the survival of Salmonella entericaon tomatoes. Int. J. Food Sci. Technol. 50, 1102-1108. doi: 10.1111/ijfs. 12748

Paniagua, A. C., East, A. R., Hindmarsh, J. P., and Heyes, J. A. (2013). Moisture loss is the major cause of firmness change during postharvest storage of blueberry. Postharv. Biol. Technol. 79, 13-19. doi: 10.1016/j.postharvbio.2012.12.016

Pao, S., and Davis, C. L. (1999). Enhancing microbiological safety of fresh orange juice by fruit immersion in hot water and chemical sanitizers. J. Food Prot. 62, 756-760. doi: 10.4315/0362-028x-62.7.756

Pao, S., Kelsey, D. F., Khalid, M. F., and Ettinger, M. R. (2007). Using aqueous chlorine dioxide to prevent contamination of tomatoes with Salmonella enterica and Erwinia carotovora during fruit washing. J. Food Prot. 70, 629-634. doi: $10.4315 / 0362-028 \mathrm{x}-70.3 .629$ 
Park, S. H., and Kang, D. H. (2015a). Antimicrobial effect of chlorine dioxide gas against foodborne pathogens under differing conditions of relative humidity. LWT Food Sci. Technol. 60, 186-191. doi: 10.1016/j.lwt.2014.09.031

Park, S. H., and Kang, D. H. (2015b). Combination treatment of chlorine dioxide gas and aerosolized sanitizer for inactivating foodborne pathogens on spinach leaves and tomatoes. Int. J. Food Microbiol. 207, 103-108. doi: 10.1016/j. ijfoodmicro.2015.04.044

Park, S. H., and Kang, D. H. (2018). Effect of temperature on chlorine dioxide inactivation of Escherichia coli O157:H7, Salmonella typhimurium, and Listeria monocytogenes on spinach, tomatoes, stainless steel, and glass surfaces. Int. J. Food Microbiol. 275, 39-45. doi: 10.1016/j.ijfoodmicro.2018.03.015

Popa, I., Hanson, E. J., Todd, E. C., Schilder, A. C., and Ryser, E. T. (2007). Efficacy of chlorine dioxide gas sachets for enhancing the microbiological quality and safety of blueberries. J. Food Prot. 70, 2084-2088. doi: 10.4315/0362-028x-70.9. 2084

Praeger, U., Herppich, W. B., and Hassenberg, K. (2018). Aqueous chlorine dioxide treatment of horticultural produce: Effects on microbial safety and produce quality-A review. Crit. Rev. Food Sci. Nutr. 58, 318-333. doi: 10.1080/10408398. 2016.1169157

Ray, S., Jin, T., Fan, X., Liu, L., and Yam, K. L. (2013). Development of chlorine dioxide releasing film and its application in decontaminating fresh produce. J. Food Sci. 78, M276-M284. doi: 10.1111/1750-3841.12010

Rodgers, S. L., Cash, J. N., Siddiq, M., and Ryser, E. T. (2004). A comparison of different chemical sanitizers for inactivating Escherichia coli O157:H7 and Listeria monocytogenes in solution and on apples, lettuce, strawberries, and cantaloupe. J. Food Prot. 67, 721-731. doi: 10.4315/0362-028x-67.4.721

Sharma, V. K., and Sohn, M. (2012). Reactivity of chlorine dioxide with amino acids, peptides, and proteins. Environ. Chem. Lett. 10, 255-264. doi: 10.1007/ s10311-012-0355-5

Simonet, J., and Gantzer, C. (2006). Degradation of the Poliovirus 1 genome by chlorine dioxide. J. Appl. Microbiol. 100, 862-870. doi: 10.1111/j.1365-2672. 2005.02850.x

Singh, N., Singh, R. K., Bhunia, A. K., and Stroshine, R. L. (2002). Effect of inoculation and washing methods on the efficacy of different sanitizers against Escherichia coli O157:H7 on lettuce. Food Microbiol. 19, 183-193. doi: 10.1006/ fmic.2001.0471

Singh, S., Maji, P. K., Lee, Y. S., and Gaikwad, K. K. (2021). Applications of gaseous chlorine dioxide for antimicrobial food packaging: a review. Environ. Chem. Lett. 19, 253-270. doi: 10.1007/s10311-020-01085-8

Song, H.-J., Choi, D.-W., and Song, K. B. (2011). Effect of aqueous chlorine dioxide and UV-C treatment on the microbial reduction and color of cherry tomatoes. Horticult. Environ. Biotechnol. 52, 488-493. doi: 10.1007/s13580-011-0043-6

Sun, X., Bai, J., Ference, C., Wang, Z., Zhang, Y., Narciso, J., et al. (2014). Antimicrobial activity of controlled-release chlorine dioxide gas on fresh blueberries. J. Food Prot. 77, 1127-1132. doi: 10.4315/0362-028X.JFP-13-554

Sun, X., Baldwin, E., and Bai, J. (2019). Applications of gaseous chlorine dioxide on postharvest handling and storage of fruits and vegetables - A review. Food Control 95, 18-26. doi: 10.1016/j.foodcont.2018.07.044

Sun, X., Baldwin, E., Plotto, A., Narciso, J., Ference, C., Ritenour, M., et al. (2017b). Controlled-release of Chlorine Dioxide in a Perforated Packaging System to Extend the Storage Life and Improve the Safety of Grape Tomatoes. J. Vis. Exp. 2017:55400. doi: 10.3791/55400

Sun, X., Baldwin, E., Ference, C., Narciso, J., Plotto, A., Ritenour, M., et al. (2017a). The Effect of Controlled-release Chlorine Dioxide on the Preservation of Grapefruit. HortScience 52, 122-126. doi: 10.21273/hortsci11363-16

Sun, X., Bin, Z., Yaguang, L., Christopher, F., Elizabeth, B., Ken, H., et al. (2017c). Effect of controlled-release chlorine dioxide on the quality and safety of cherry/grape tomatoes. Food Control 82, 26-30. doi: 10.1016/j.foodcont.2017. 06.021

Sy, K. V., McWatters, K. H., and Beuchat, L. R. (2005a). Efficacy of gaseous chlorine dioxide as a sanitizer for killing Salmonella, yeasts, and molds on blueberries, strawberries, and raspberries. J. Food Prot. 68, 1165-1175. doi: 10.4315/0362$028 \mathrm{x}-68.6 .1165$

Sy, K. V., Murray, M. B., Harrison, M. D., and Beuchat, L. R. (2005b). Evaluation of gaseous chlorine dioxide as a sanitizer for killing Salmonella, Escherichia coli O157:H7, Listeria monocytogenes, and yeasts and molds on fresh and fresh-cut produce. J. Food Prot. 68, 1176-1187. doi: 10.4315/0362-028x-68.6.1176
Trinetta, V., Linton, R. H., and Morgan, M. T. (2013). Use of chlorine dioxide gas for the postharvest control of Alternaria alternata and Stemphylium vesicarium on Roma tomatoes. J. Sci. Food Agric. 93, 3330-3333. doi: 10.1002/jsfa.6180

Trinetta, V., Morgan, M. T., and Linton, R. H. (2010). Use of high-concentrationshort-time chlorine dioxide gas treatments for the inactivation of Salmonella enterica spp. inoculated onto Roma tomatoes. Food Microbiol. 27, 1009-1015. doi: 10.1016/j.fm.2010.06.009

Trinetta, V., Vaidya, N., Linton, R., and Morgan, M. (2011). Evaluation of chlorine dioxide gas residues on selected food produce. J. Food Sci. 76, T11-T15. doi: 10.1111/j.1750-3841.2010.01911.x

Wang, Z., Narciso, J., Biotteau, A., Plotto, A., Baldwin, E., and Bai, J. (2014). Improving Storability of Fresh Strawberries with Controlled Release Chlorine Dioxide in Perforated Clamshell Packaging. Food Bioproc. Technol. 7, 35163524. doi: 10.1007/s11947-014-1364-0

WHO (2015). WHO estimates of the global burden of foodborne diseases: foodborne disease burden epidemiology reference group 2007-2015. Geneva: WHO.

Wu, V. C., and Kim, B. (2007). Effect of a simple chlorine dioxide method for controlling five foodborne pathogens, yeasts and molds on blueberries. Food Microbiol. 24, 794-800. doi: 10.1016/j.fm.2007.03.010

Wu, V. C., and Rioux, A. (2010). A simple instrument-free gaseous chlorine dioxide method for microbial decontamination of potatoes during storage. Food Microbiol. 27, 179-184. doi: 10.1016/j.fm.2009.08.007

Xue, B., Jin, M., Yang, D., Guo, X., Chen, Z., Shen, Z., et al. (2013). Effects of chlorine and chlorine dioxide on human rotavirus infectivity and genome stability. Water Res. 47, 3329-3338. doi: 10.1016/j.watres.2013.03.025

Yoon, J. H., and Lee, S. Y. (2018). Review: Comparison of the Effectiveness of Decontaminating Strategies for Fresh Fruits and Vegetables and Related Limitations. Crit. Rev. Food Sci. Nutr. 58, 3189-3208. doi: 10.1080/10408398. 2017.1354813

Young, S. B., and Setlow, P. (2003). Mechanisms of killing of Bacillus subtilis spores by hypochlorite and chlorine dioxide. J. Appl. Microbiol. 95, 54-67. doi: 10.1046/j.1365-2672.2003.01960.x

Zhang, B., Huang, C., Zhang, L., Wang, J., Huang, X., Zhao, Y., et al. (2019). Application of chlorine dioxide microcapsule sustained-release antibacterial films for preservation of mangos. J. Food Sci. Technol. 56, 1095-1103. doi: 10.1007/s13197-019-03636-6

Zhang, X. M., and Fu, M. R. (2018). Inhibitory effect of chlorine dioxide $\left(\mathrm{ClO}_{2}\right)$ fumigation on growth and patulin production and its mechanism in Penicillum expansum. LWT 96, 335-343. doi: 10.1016/j.lwt.2018.05.051

Zhou, S., Hu, C., Zhao, G., Jin, T., Sheen, S., Han, L., et al. (2018). Novel generation systems of gaseous chlorine dioxide for Salmonella inactivation on fresh tomato. Food Control 92, 479-487. doi: 10.1016/j.foodcont.2018. 05.025

Zhu, C., Chen, Z., and Yu, G. (2013). Fungicidal mechanism of chlorine dioxide on Saccharomyces cerevisiae. Ann. Microbiol. 63, 495-502. doi: 10.1007/s13213012-0494-8

Zhu, Z., Guo, Y., Yu, P., Wang, X., Zhang, X., Dong, W., et al. (2019). Chlorine dioxide inhibits the replication of porcine reproductive and respiratory syndrome virus by blocking viral attachment. Infect. Genet. Evol. 67, 78-87. doi: 10.1016/j.meegid.2018.11.002

Conflict of Interest: The authors declare that the research was conducted in the absence of any commercial or financial relationships that could be construed as a potential conflict of interest.

Publisher's Note: All claims expressed in this article are solely those of the authors and do not necessarily represent those of their affiliated organizations, or those of the publisher, the editors and the reviewers. Any product that may be evaluated in this article, or claim that may be made by its manufacturer, is not guaranteed or endorsed by the publisher.

Copyright (c) 2022 Malka and Park. This is an open-access article distributed under the terms of the Creative Commons Attribution License (CC BY). The use, distribution or reproduction in other forums is permitted, provided the original author(s) and the copyright owner(s) are credited and that the original publication in this journal is cited, in accordance with accepted academic practice. No use, distribution or reproduction is permitted which does not comply with these terms. 